\title{
Síntesis de ZnO con morfología acicular por el método de precipitación controlada (MPC) y su uso como refuerzo de elastómeros
}

\author{
E. J. ANDRADE CAMPO', H. ARISTIZABAL², J. E. RODRÍGUEZ-PÁEZ1 \\ ${ }^{1}$ Grupo CYTEMAC-Universidad del Cauca \\ Calle 5 No 4-70 Popayán Cauca / Colombia. \\ Tel 820-98-00-ext, 2410 \\ ${ }^{2}$ ICOBANDAS S. A. Cauca Alto-Popayán/Colombia.
}

\begin{abstract}
El óxido de cinc, $\mathrm{ZnO}$, es un compuesto de gran interés industrial. Se utiliza como activador en el proceso de vulcanización de los cauchos, en la industria de los pigmentos y dadas sus propiedades eléctricas, como supresor de sobre tensiones y material piezoeléctrico. Estas aplicaciones requieren que las partículas de $\mathrm{ZnO}$ presenten determinadas características: pureza química, tamaño, distribución de tamaño y morfología de las partículas. En este trabajo se realizó el estudio de la síntesis de $\mathrm{ZnO}$ por el método de precipitación controlada (MPC) para obtener partículas con morfología acicular. Los ensayos de valoración potenciométrica y conductimétrica permitieron determinar las diferentes etapas que ocurren durante la adición del agente precipitante $\left(\mathrm{NH}_{4} \mathrm{OH}\right)$ a las disoluciones acuosas de cinc y por lo tanto, permiten garantizar la reproducibilidad del método. Los compuestos obtenidos se caracterizaron utilizando espectroscopia infrarroja (FTIR), difracción de rayos X (DRX), análisis térmico (TG) y microscopia electrónica de transmisión (MET). Después de sintetizado el material, el ZnO acicular se utilizó en el proceso de vulcanización de caucho polibutadieno y se determinó su efecto sobre las propiedades de los elastómeros. Los ensayos que se utilizaron para tal fin fueron: resistencia a la tracción y al desgarro, abrasión, elongación y módulo al $100 \%$.
\end{abstract}

Palabras clave: oxido de cinc; método de coprecipitación; vulcanización; reforzante.

Synthesis of needles $\mathrm{ZnO}$ by the controlled precipitation method and its usage as a reinforcing agent for elastometer

The zinc oxide, $\mathrm{ZnO}$, is a compound of high technological interest. It is used as a catalyst in the vulcanizing process, as a pigment and due to its electrical properties as a suppressor of electrical overload and piezoelectric material. These applications require that the $\mathrm{ZnO}$ present high chemical purity and determinate morphology and size of particles. In this work we studied the $\mathrm{ZnO}$ synthesis using the controlled precipitation method to obtain needles shape particles. The potentiometer and conductimetic titration indicated the different stages that take place during the $\mathrm{NH}_{4} \mathrm{OH}$ addition to Zinc solution. Besides, these curves allow to conform the reproducilety of this method. The obtained ceramic powder was characterized by infrared spectroscopy, $X$ ray diffraction (XRD), thermal analysis (TG/DTA) and transmission electron microscopy (TEM). The synthesized $\mathrm{ZnO}$ was used the mixture and the mechanical properties of elastomeric ribbon were determinated.

Keywords: Zinc oxide; co-precipitation method; elastomer vulcanization and reinforcement.

\section{INTRODUCCIÓN}

El óxido de cinc, $\mathrm{ZnO}$, es un semiconductor con estructura tipo Wurtzita que presenta una constante dieléctrica no muy baja, un factor de potencia bajo, alta resistividad y poca adsorción de agua. Esto hace que el oxido de cinc puro, o con otros óxidos como dopantes, sea un material de interés para la industria y se utilice en la elaboración de catalizadores, dispositivos eléctricos y electrónicos (1). Además es utilizado como pigmento, en la producción de pinturas, presentando ventajas sobre el "plomo blanco" (carbonato básico de plomo) dada su baja toxicidad (2).

Debido a los requerimientos tecnológicos actuales es necesario desarrollar nuevos métodos de síntesis (no convencionales) que permitan obtener $\mathrm{ZnO}$ nanométrico con diversas morfologías entre los que se destacan los procesos sol-gel (3), Pechini (4), e hidrotermal; utilizando este último proceso Wen-Jun $\mathrm{Li}$ y colaboradores obtuvieron $\mathrm{ZnO}$ con morfología acicular (5).

En este trabajo se utilizó el método de precipitación controlada (MPC), para sintetizar el $\mathrm{ZnO}$ acicular, el cual ha sido utilizado con éxito para obtener óxidos de interés industrial (6 - 9). En este método se parte de la disolución de una sal en un medio acuoso, acidulado o no, a la que se le adiciona controladamente un agente precipitante. Los cambios de $\mathrm{pH}$, conductividad específica y temperatura, así como las modificaciones que visualmente se observan en el sistema, permiten conocer los cambios del mismo y por lo tanto 
controlar lo que en él ocurre (10).

Se analizaron los principales fenómenos fisicoquímicos que ocurren en el sistema durante la síntesis del $\mathrm{ZnO}$, con morfología acicular, y se caracterizó el polvo cerámico obtenido mediante difracción de rayos-X (DRX), espectroscopia infrarroja (FT-IR) y microscopía electrónica de transmisión (MET). El óxido de cinc con morfología acicular se utilizó en el conformado de elastómeros y a través de los ensayos de resistencia a la tracción y al desgarro, elongación y módulo al $100 \%$, del vulcanizado, se determinó el poder reforzante de este óxido.

\section{PROCESO EXPERIMENTAL}

\subsection{Síntesis del $\mathrm{ZnO}$ acicular dada su morfología.}

Para la obtención del oxido de cinc con morfología acicular se empleó como precursor de cinc el acetato de cinc ( $\mathrm{Zn}\left(\mathrm{CH}_{3} \mathrm{COO}\right)_{2}$-Aldrich), en una concentración $0.34 \mathrm{M}$, disuelto en $200 \mathrm{~mL}$ de agua destilada; la solución se aciduló con ácido nítrico $\mathrm{HNO}_{3}$ (Carlo Erba Reagenti) $0.1 \mathrm{~N}$, posteriormente se agregó regularmente volúmenes iguales de hidróxido de amonio, $\mathrm{NH}_{4} \mathrm{OH}$ (Mallinckrodt 28.0-30.0\%), por medio de un dosimat Metrohm 775, a la solución en continua agitación a $400 \mathrm{rpm}$.

Durante la adición del $\mathrm{NH}_{4} \mathrm{OH}$ (agente precipitante) se registraron simultáneamente el valor del $\mathrm{pH}$, utilizando un pH-metro Metrohm 744, y la conductividad específica, mediante un conductímetro Metrohm 722, lo que permitió determinar el avance de las reacciones químicas en la solución y generar un medio de control sobre el sistema a través de estos parámetros.

La suspensión se dejó en reposo durante 24 horas, a temperatura ambiente, luego se filtró al vacío y el sólido húmedo obtenido se redispersó en agua destilada utilizando un equipo de alta cizalla ultra-turrax IKA T-50. El mismo proceso se repitió dos veces más y finalmente la muestra se secó a una temperatura de $80^{\circ} \mathrm{C}$; a este conjunto de actividades se le denominó proceso de "lavado".

Posteriormente, las muestras sólidas se maceraron en un mortero ágata y se caracterizaron con difracción de rayos-X (DRX), utilizando el difractómetro Siemens D-5000Erlangen Germany, espectroscopia infrarroja (FT-IR), con un espectrofotómetro marca Shimadzu FT-IR con transformada de Fourier, y microscopia electrónica de transmisión (MET) empleando un equipo JEOL 1200 EX (a $80 \mathrm{Kev}$ ).

\section{2 formulación de mezclas de caucho}

La conformación de la mezcla de caucho se realizó en la planta de ICOBANDAS S.A., Popayán-Colombia, empleándose para su formulación 100 phr (peso en 100 partes de caucho) de caucho polibutadieno, con el cual se obtiene un producto vulcanizado de alta resistencia a la abrasión y flexibilidad a bajas temperaturas.

Para ello, el caucho se alimentó a temperatura ambiente y se masticó de 4 a 5 minutos en un molino mezclador abierto, hasta alcanzar una temperatura entre 50 y $60^{\circ} \mathrm{C}$, para obtener una muy buena incorporación de las sustancias en la mezcla. Una vez homogenizado el caucho, este se tornó pegajoso y adquirió un estado plástico, facilitándose la incorporación de otras sustancias tales como pigmentos, agentes vulcanizantes, cargas reforzantes, agentes protectores etc., que se utilizaron para modificar sus propiedades finales.

Cuando el caucho estuvo totalmente homogéneo se adicionaron todas las sustancias excepto los acelerantes, y se inició el proceso de incorporación, operación que demoró entre 6 y 8 minutos. Por último se adicionaron los acelerantes y se continúo con el proceso de homogeneización final que duró entre 2 y 4 minutos. Incorporadas todas las sustancias, la mezcla se laminó a un espesor aproximado de $7 \mathrm{~mm}$, a una temperatura final de $80{ }^{\circ} \mathrm{C}$, y se sumergió en una solución antiadherente para realizar el enfriamiento y evitar la vulcanización prematura. Las condiciones de vulcanización que se utilizaron fueron: temperatura $150^{\circ} \mathrm{C}$, presión de 20 $\mathrm{KgF} / \mathrm{cm}^{2}$ y tiempo de vulcanización 15 minutos.

Posteriormente, de la lámina fría, se conformaron las probetas que se utilizaron para realizar los diferentes ensayos de caracterización mecánica de la lámina de caucho Para ello se realizaron ensayos de abrasión, dureza, tracción, desgarro, elongación, adhesión y módulo al 100\% (11), ensayos que se realizaron en la planta de ICOBANDAS S.A. de acuerdo con las normas ASTM (12).

\section{RESULTADOS}

\subsection{Curvas de valoración potenciométrica y conductimétrica}

En la figura 1 se muestran las curvas de valoración potenciométrica y conductimétrica del sistema acuoso de acetato de cinc. Según la literatura $(10,13)$ en la solución inicial de $\mathrm{Zn}\left(\mathrm{CH}_{3} \mathrm{COO}\right)_{2}$, previo a la adición de $\mathrm{NH}_{4} \mathrm{OH}$, las principales especies que se conforman son complejos acuo de los siguientes iones: $\mathrm{Zn}^{+2}, \mathrm{CH}_{3} \mathrm{COO}, \mathrm{Zn}\left(\mathrm{CH}_{3} \mathrm{COO}\right)\left(\mathrm{OH}_{2}\right)_{n^{\prime}}$ $\mathrm{H}_{3} \mathrm{O}^{+}$y OH . La naturaleza del precursor de cinc debe favorecer la formación del complejo $\left[\mathrm{Zn}\left(\mathrm{OH}_{2}\right)_{6}\right]^{2+}$, existiendo además especies hidroxo $\left[\mathrm{Zn}(\mathrm{OH})_{4}\right]^{2-}$ cuya estabilidad se justifica a través de una serie de reacciones que se indican más adelante y que se ve favorecida porque el $\mathrm{Zn}$ disuelto en una base tiene la tendencia a formar iones cincato tales como (14): $\left[\mathrm{Zn}(\mathrm{OH})_{3}\left(\mathrm{H}_{2} \mathrm{O}\right)\right]^{-},\left[\mathrm{Zn}(\mathrm{OH})_{3}\left(\mathrm{H}_{2} \mathrm{O}\right)_{3}\right]^{-}$o $\left[\mathrm{Zn}(\mathrm{OH})_{4}\right]^{2-}$.

$\mathrm{Al}$ adicionar el $\mathrm{NH}_{4} \mathrm{OH}$, a la solución de acetato de cinc, pueden ocurrir reacciones que producen complejos de acetatoamónico del tipo $\mathrm{Zn}\left(\mathrm{CH}_{3} \mathrm{COO}\right)_{\mathrm{x}}\left(\mathrm{NH}_{3}\right)_{\mathrm{y}}{ }^{(2-\mathrm{x})+}(8,9)$. Como el $\mathrm{Zn}^{2+}$ es un catión tipo $\mathrm{B}$, más afín al nitrógeno, al adicionarse el $\mathrm{NH}_{4}^{+}$a la solución el ligando acuo se desplazaría de la esfera de coordinación del $\mathrm{Zn}^{2+}$, favoreciéndose la formación de un acetato básico de cinc de acuerdo a la siguiente reacción (15):

$\mathrm{Zn}\left(\mathrm{CH}_{3} \mathrm{COO}\right)_{2}+\mathrm{nNH}_{4} \mathrm{OH} \quad \mathrm{Zn}(\mathrm{OH})_{\mathrm{n}}\left(\mathrm{CH}_{3} \mathrm{COO}\right)_{2-\mathrm{n}} \mathrm{E}+\mathrm{nNH}_{4} \mathrm{CH}_{3} \mathrm{COO}$

La curva de valoración potenciométrica de la figura 1, correspondiente al sistema $0.34 \mathrm{M}$ de $\mathrm{Zn}\left(\mathrm{CH}_{3} \mathrm{COO}\right)_{2} 2 \mathrm{H}_{2} \mathrm{O}$, muestra claramente tres (3) zonas que se pueden definir con base en los cambios de pendiente que ella presenta. La presencia del $\mathrm{HNO}_{3}$ afecta principalmente la zona 1 donde el $\mathrm{NH}_{4} \mathrm{OH}$ que se adicionó permitió neutralizar el ácido fuerte existente en el sistema. Una reacción importante que debe ocurrir durante las primeras etapas del proceso, disolución de la sal y zona 1 de la curva de valoración potenciométrica, es la relacionada con la formación de un nitrato básico a través de la siguiente reacción: 
$\mathrm{Zn}\left(\mathrm{CH}_{3} \mathrm{COO}\right)_{2} 2 \mathrm{H}_{2} \mathrm{O}+2 \mathrm{HNO}_{3} \quad \mathrm{Zn}\left(\mathrm{NO}_{3}\right)_{2}\left(\mathrm{OH}_{2}\right)_{\mathrm{n}}+2 \mathrm{CH}_{3} \mathrm{COOH}$

reacción que se justifica por la afinidad que presenta el $\mathrm{Zn}^{2+}$ por el nitrógeno, lo que ocasionaría el desplazamiento de los demás ligandos. La presencia del ion $\mathrm{NO}_{3}^{-}$en el sistema favorecería la formación de la especie polinuclear $\mathrm{Zn}_{5}\left(\mathrm{NO}_{3}\right)_{2}(\mathrm{OH})_{8} .2 \mathrm{NH}_{3}$ durante el secado debido a que en el sistema se precipita $\mathrm{Zn}_{5}(\mathrm{OH})_{8}\left(\mathrm{NO}_{3}\right)_{2}(8,9)$.

Además, dado que en el sistema existen los compuestos $\mathrm{Zn}_{5}(\mathrm{OH})_{8}\left(\mathrm{NO}_{3}\right)$ y $\mathrm{Zn}\left(\mathrm{CH}_{3} \mathrm{COO}\right)_{2}$, se favorece la conformación del acetato básico de cinc en la suspensión coloidal, tal como sucede con los compuestos sintetizados por Kawai y colaboradores (15).

Como se observa en la curva de valoración potenciométrica de la figura 1, esta alcanza una condición de saturación y experimentalmente se observa que la suspensión comienza a redisolverse produciéndose el complejo de cinc $\mathrm{Zn}\left(\mathrm{CH}_{3} \mathrm{COO}\right)_{2}(\mathrm{n}+\mathrm{x}) \mathrm{NH}_{3}(8,9)$.

Con respecto a la curva de valoración conductimétrica, figura 1, obtenida para la solución de $\mathrm{Zn}\left(\mathrm{CH}_{3} \mathrm{COO}\right)_{2^{\prime}}$ se observa en ella regiones donde la conductividad especifica varía linealmente con el volumen de base adicionada; la curva presenta la forma típica de una valoración de ácido débil con una base débil. El valor de la conductividad se va incrementando en el primer trayecto por la formación progresiva de complejos de cinc en la disolución del sistema y la saturación se alcanza en el segundo tramo a valores altos de $\mathrm{pH}$ ya que el $\mathrm{NH}_{4} \mathrm{OH}$ adicionado al sistema está poco ionizado. La acción reguladora del sistema se pone en evidencia en esta segunda región de la curva donde la ionización del ácido débil se desarrolla lentamente lo que ocasiona que el valor de la conductividad permanezca constante.

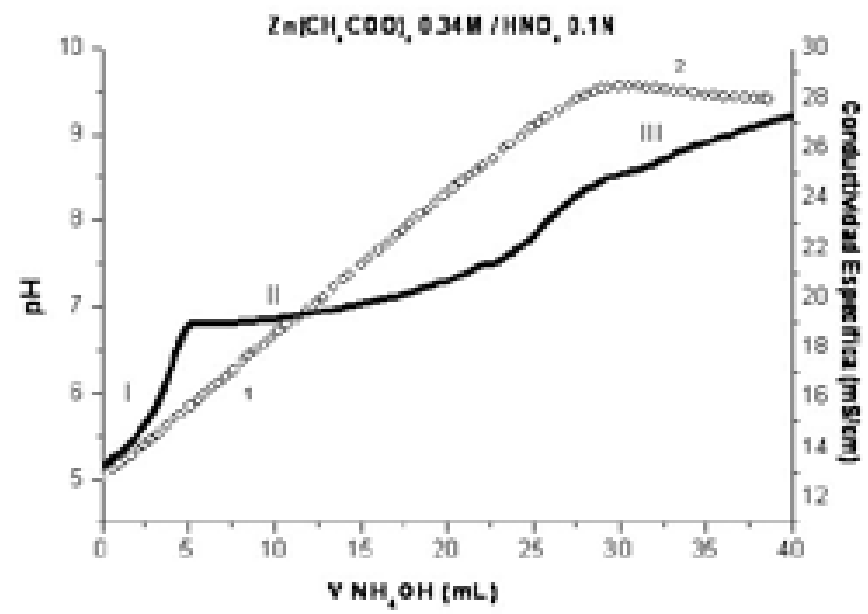

Fig. 1- Curva de valoración potenciomentrica y conductimetrica de solución 0.34M de acetato de cinc.

\subsection{Caracterización con espectroscopia infrarroja (FT-IR)}

En la figura 2 se presentan los espectros FT-IR del sólido obtenido a $\mathrm{pH} 8.4$ y sometido a diferentes etapas del proceso de lavado. Las bandas anchas entre $\sim 3000 \mathrm{~cm}^{-1}$ y $3200 \mathrm{~cm}^{-1}$ contienen los modos vibracionales de tensión correspondientes a los grupos funcionales $\mathrm{O}-\mathrm{H}$ y N-H, siendo más evidentes las que se asocian al enlace $\mathrm{N}-\mathrm{H}$ del $\mathrm{NH}_{3}$ físicamente adsorbido. La banda a $\sim 3337 \mathrm{~cm}^{-1}$ está indicando el enlace que establece el $\mathrm{NH}_{4}^{+}$con un ión menos polarizable.

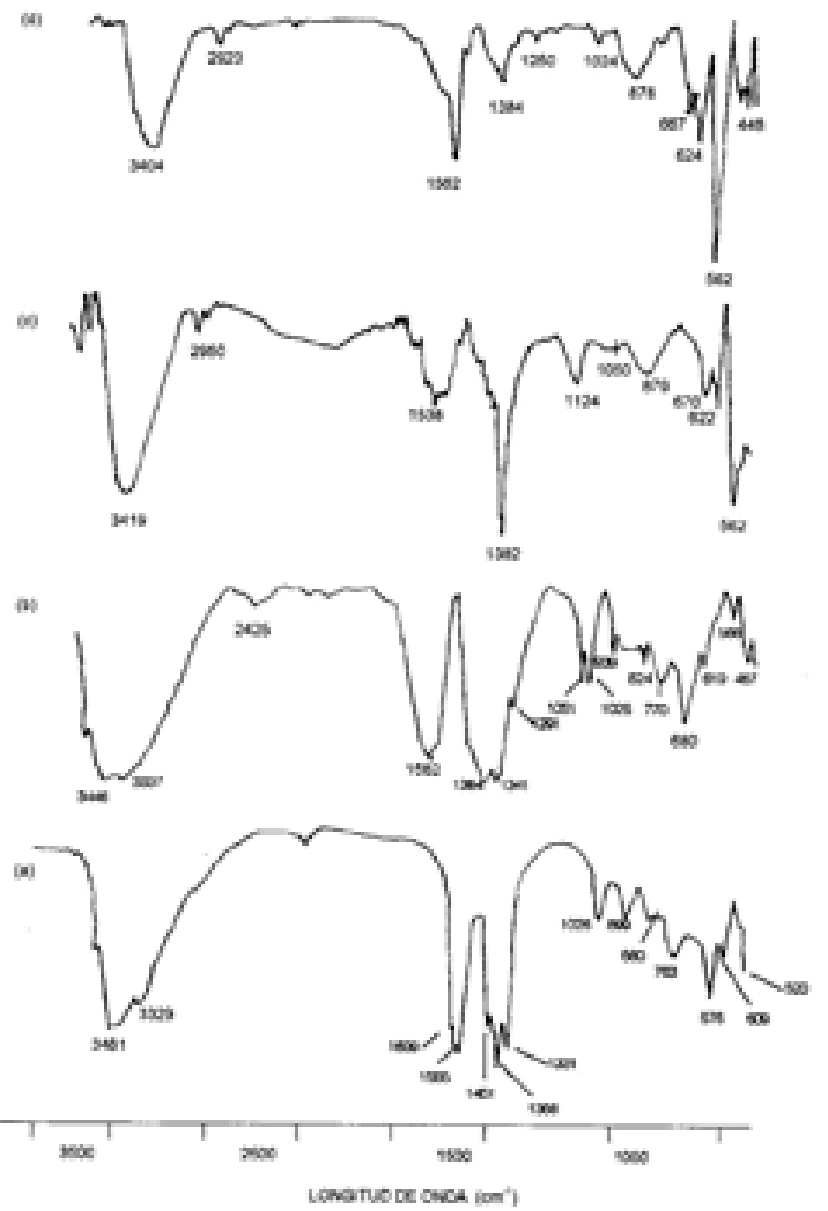

Fig. 2- Espectro infrarrojo de muestras sólidas de acetato de cinc obtenidas a pH 8.4: (a) Sin lavado, (b) primer lavado, (c) segundo lavado, (d) tercer lavado.

Las bandas entre $3404 \mathrm{~cm}^{-1}$ y $3327 \mathrm{~cm}^{-1}$ también están asociadas al $\mathrm{NH}_{3}$ (16). En la zona entre $1500 \mathrm{~cm}^{-1}$ y $1200 \mathrm{~cm}^{-1}$ se observan grandes cambios en la intensidad de las bandas durante el proceso de lavado. Las bandas a $1582 \mathrm{~cm}^{-1}$ y 1341 $\mathrm{cm}^{-1}$, se pueden asignar a los modos vibracionales de tensión $v_{\text {as }}$ $(\mathrm{COO}) \mathrm{y}_{\mathrm{s}}(\mathrm{COO})$, que presentan una separación de frecuencia de $\Delta v=241 \mathrm{~cm}^{-1}$, indicando la formación de un compuesto monodentado con el carboxilo. Pero, considerando las bandas anchas alrededor de $1582 \mathrm{~cm}^{-1}$ y entre $1384 \mathrm{~cm}^{-1}$ y $1341 \mathrm{~cm}^{-1}$, estas pueden contener información adicional de la presencia de especies bidentadas quelante y puente del carboxilo; en el sistema existen, por lo tanto, especies monodentadas y bidentadas.

En el espectro de la muestra del segundo lavado aparecen bandas a $1538 \mathrm{~cm}^{-1}$ y $1382 \mathrm{~cm}^{-1}$, con una separación de frecuencia de $\Delta v=156 \mathrm{~cm}^{-1}$, indicando la presencia de un enlace bidentado del grupo carboxilo. La muestra correspondiente al tercer lavado presenta una separación de las bandas, asociadas al grupo funcional COO, de $\Delta v=198 \mathrm{~cm}^{-1}$ ya que el $v_{\text {as }}$ se puede asociar a la banda ubicada a $1582 \mathrm{~cm}^{-1}$ y el $v_{\mathrm{s}}$ a la que se encuentra a $1384 \mathrm{~cm}^{-1}$ esto indica que el ion acetato se coordina al metal conformando una estructura puente y/o monodentada a medida que se avanza en el proceso de lavado (17). La banda a $1124 \mathrm{~cm}^{-1}$ corresponde al modo v(CO), y la ubicada a $622 \mathrm{~cm}^{-1}$ se puede asociar a los modos $\pi(\mathrm{COO})$ $+\pi(\mathrm{CH})$. En la zona entre $1000 \mathrm{~cm}^{-1}$ y $400 \mathrm{~cm}^{-1}$ se presentan 
cambios muy evidentes en el espectro, con aparición de nuevas bandas así como con el aumento en la intensidad de las existentes a medida que se avanza en el proceso de lavado. Las bandas a $\sim 562 \mathrm{~cm}^{-1}$, segundo y tercer lavado, y $446 \mathrm{~cm}^{-1}$, para el tercer lavado, indican de manera evidente la presencia del enlace Zn-O; el desplazamiento de estas bandas de absorción se pude justificar con base en la evolución de la morfología de las partículas, tal como se observa en las fotografías de MET tomadas a las muestras estudiadas.

\section{Caracterización con difracción de rayos- $x$ (DRX)}

En la figura 3 se muestran los difractogramas de rayos $x$ de sólidos del sistema de acetato obtenidos a medida que se avanza en el proceso de lavado. En el difractograma de la figura 3(b) empiezan a ser evidentes los picos de difracción característicos del ZnO (PDF 36 1451), además de los que corresponden a las siguientes fases cristalinas: $\mathrm{Zn}(\mathrm{OH})_{2}(\mathrm{PDF}$ 48 1066) y $\mathrm{Zn}\left(\mathrm{NO}_{3}\right)_{2}(\mathrm{OH})_{8} 2 \mathrm{NH}_{3}$ (PDF 45-5930). Las fases presentes en el sólido correspondiente al segundo lavado son: $\mathrm{ZnO}$ (PDF 79 2205) y el Zn (OH) (PDF 48 1066). En la muestra del tercer lavado están presentes las fases: $\mathrm{ZnO}$ (PDF 79 2205), $\mathrm{Zn}(\mathrm{OH})_{2}$ (PDF 48 1066) y $\mathrm{Zn}_{5}\left(\mathrm{NO}_{3}\right)(\mathrm{OH})_{8} 2 \mathrm{NH}_{3}$ (PDF 45-5930).

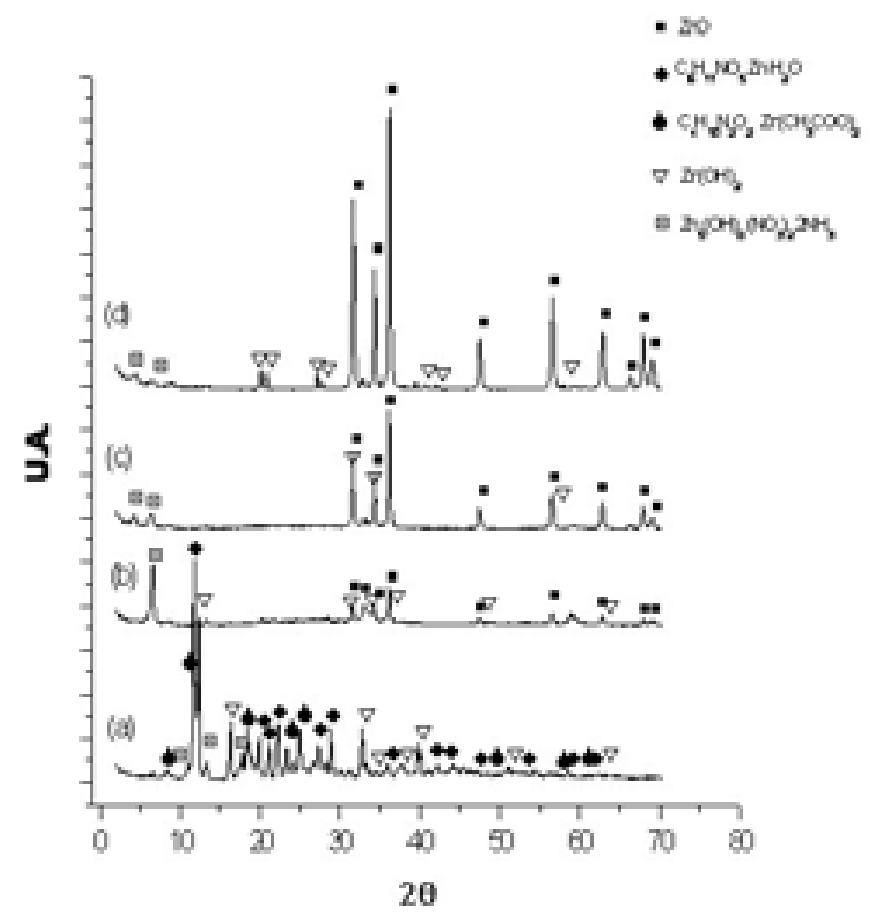

Fig. 3- Difractograma de rayos $x$ de muestras sólidas obtenidas del sistema de acetato de cinc $0.34 \mathrm{M}$ a pH $8.4 ;$ y que fueron sometidos al proceso de lavado: (a) sin lavado, (b) primer lavado, (c) segundo lavado y (d) tercer lavado.

La formación del $\mathrm{ZnO}$ en el sólido, durante el proceso de lavado, se ve favorecida por el efecto que brinda la acción de cizalla para inducir descomposiciones químicas y/o transformaciones, y que es generada durante la redispersión del sedimento al utilizar el ultraturrax a $10000 \mathrm{rpm}$, así como la interacción solvente - soluto que ocasiona desgaste químico de las nanoparticulas y re-cristalización de las mismas.

\section{Microscopia electrónica de transmisión (MET)}

En la figura 4 se ilustra la evolución de la morfología de las partículas al someter la suspensión obtenida a $\mathrm{pH} 8.4$ al proceso de lavado. La figura 4 (a) muestra la morfología de las partículas que resultan del primer lavado; el polvo presenta gran aglomeración con un tamaño de $\sim 1 \mu \mathrm{m}$. La fotografía de la figura 4 (b) corresponde a partículas del segundo lavado algunas de las cuales ya presentan morfología acicular; la aglomeración es menor y la longitud de partícula es de $\sim 1$ $\mu \mathrm{m}$. En la muestra del tercer lavado, las partículas presentan claramente la forma acicular, con un tamaño nanométrico, aunque la longitud de las pequeñas fibras es del orden de las micras. Según el difractograma de rayos $x$, figura 3(d), ésta muestra contiene $\mathrm{ZnO}$ como fase mayoritaria.
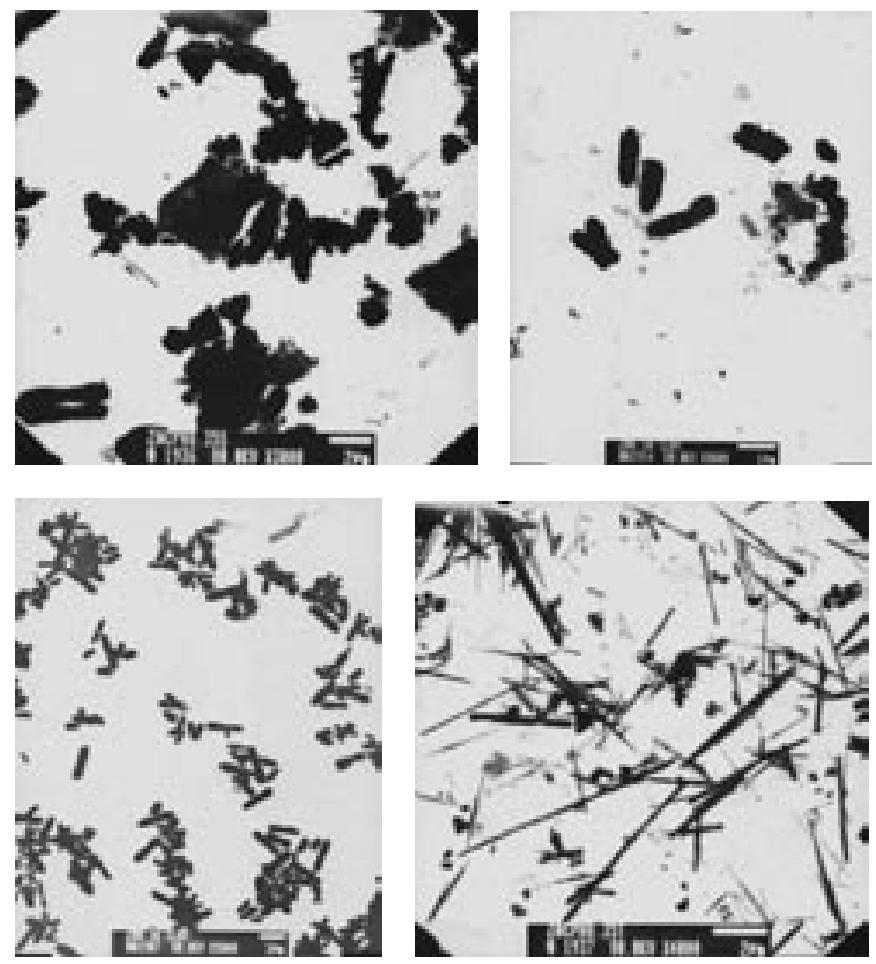

Fig. 4- Fotografías obtenidas de MET de muestras sólidas del sistema acetato de cinc $0.34 \mathrm{M}$, a pH 8.4 sometidas a proceso de lavado: (a) sin lavado, (b) primer lavado, (c) segundo lavado y (d) tercer lavado.

\section{Características mecánicas de las mezclas de caucho}

Con el fin de realizar una adecuada caracterización mecánica de las mezclas de caucho y obtener resultados representativos y fiables, dada la cantidad de muestras y los diversos parámetros que entran en juego, se utilizó un método estadístico de diseño de experimentos. Este modelo estadístico buscaba determinar los factores que influían sobre la variable de interés y si existían influencias cuantificarlas. El método Taguchi (18), utilizado en el este trabajo, hace uso de factoriales con factores a tres niveles para garantizar procesos de calidad y reducción de costos emanados por la experimentación; este es el método que se emplea normalmente en la industria del caucho.

El número de variables que se analizaron fueron dos: la primera variable tiene que ver con el origen del $\mathrm{ZnO}$ y la segunda variable con la cantidad de $\mathrm{SiO}_{2}$ utilizado. Con estas 
variables se realizaron 4 ensayos en un diseño factorial. Para evitar trabajar con las variables independientes, se introdujo el concepto de nivel el cual se codificó con valores arbitrarios, tal como se indica en la tabla 1 donde phr significa peso en 100 partes de caucho.

TABLA I. NUMERO DE VARIABLES Y DE FACTORES

\begin{tabular}{|c|c|c|}
\hline FACTOR & NIVEL (-) & NIVEL (+) \\
\hline $\mathrm{ZnO}$ & INDUSTRIAL & SINTETIZADO \\
$\mathrm{SiO}_{2}$ & $15 \mathrm{phr}^{*}$ & $50 \mathrm{phr}^{*}$ \\
\hline
\end{tabular}

* phr peso en 100 partes de caucho

En la tabla 2 se indica el diseño factorial de acuerdo al método Taguchi (18), donde se señala el numero de variables y las respuestas según el efecto de esta variable y su interacción, utilizando para ello el signo "-" que representa el nivel (-) y el signo " + " el nivel $(+)$. Además, se indica la interacción y la asignación de los factores en columna. De acuerdo a esta información, el diseño de experimentos proporcionó un número determinado de mezclas que permitieron, una vez realizados los ensayos de las propiedades analizar y hacer cálculos con el fin de optimizar el proceso de conformado de elastómeros. Los cálculos detallados se indican en la referencia (10).

TABLA II. ASIGNACIÓN DE FACTORES EN COLUMNA

\begin{tabular}{|c|c|c|c|}
\hline $\begin{array}{c}\text { NUMERO DEL } \\
\text { ENSAYO }\end{array}$ & $\mathrm{ZnO}$ & $\mathrm{SiO}_{2}$ & $\begin{array}{c}\text { INTERACCIÓN } \\
1 \& 2\end{array}$ \\
\hline 1 & - & - & - \\
2 & - & + & + \\
3 & + & - & + \\
4 & + & + & - \\
\hline
\end{tabular}

Para realizar los ensayos mecánicos, a las láminas obtenidas de la vulcanización de las mezclas de caucho que se estudiaron, se conformaron probetas especialmente moldeadas para dicho fin. Existen varios métodos de ensayo para determinar la calidad de los productos de caucho pero el comportamiento a la tracción es sin duda uno de los más importantes y el más utilizado a nivel industrial. El valor del esfuerzo para cada incremento en la elongación, y en el punto de rotura, es calculado sobre la base del área de la sección transversal original de la probeta. De los resultados obtenidos, referencia 10, se encontró, de acuerdo al efecto de los factores (tabla 2) sobre el comportamiento en tracción, que el mayor valor se registro para el $\mathrm{SiO}_{2}$ (372.42) y el menor valor para la interacción entre los factores (154.9). En los mismos resultados se pudo observar un incremento en la respuesta a la tracción para los diferentes ensayos destacándose un incremento del $74 \%$ al comparar el ensayo 1 con el 3 donde se varió la procedencia del $\mathrm{ZnO}$ y se mantuvo constante la calidad del $\mathrm{SiO}_{2}$. Al comparar el ensayo 2 con el ensayo 4, el incremento fue solo del $1 \%$ en ellos se aumentó la cantidad de $\mathrm{SiO}_{2}$, no así la de $\mathrm{ZnO}$, y por ello no se pudo apreciar la influencia del $\mathrm{ZnO}$. En la tabla 3 se presenta la respuesta a la tracción de acuerdo al efecto de nivel (10) observando un importante incremento en el efecto cuando se agrega el $\mathrm{ZnO}$ sintetizado, y que tiene una morfología acicular, en lugar de $\mathrm{ZnO}$ industrial que no presenta una morfología definida. Igualmente se presenta una respuesta favorable al incrementar el $\mathrm{SiO}_{2}$ de $15 \mathrm{phr}$ a 50 phr.

La fuerza necesaria para producir una elongación dada en la probeta de elastómero (modulo del material), y por lo tanto el valor del esfuerzo para ocasionar cada incremento de deformación, es también una característica importante. En la tabla 3 se muestran las respuestas de elongación y modulo al $100 \%$ con base en los resultados del análisis estadístico de los datos de los diferentes ensayos (10). Se observa para ambos casos un incremento muy favorable al utilizar el $\mathrm{ZnO}$ sintetizado.

Para el ensayo de desgarro se realizó un procedimiento de análisis de resultados similar al de tracción, donde los resultados indican poca contribución del $\mathrm{ZnO}$ (ver referencia 10). La tabla 3 muestra que el $\mathrm{ZnO}$ sintetizado, presenta un efecto ligeramente favorable si se compara con el $\mathrm{ZnO}$ industrial; el mayor incremento en el efecto se nota cuando se pasa de 15 phr a 50 phr de $\mathrm{SiO}_{2}$.

TABLA III. RESULTADO DE LOS DIFERENTES ENSAYOS PARA EL EFECTO DE NIVEL

\begin{tabular}{|c|c|c|c|c|c|c|c|}
\hline & FACTOR & NIVEL & TRACCIÒN & ELONGACION & MODULO AL $100 \%$ & DESGARRO & ABRASION \\
\hline \multirow[t]{3}{*}{1} & $\mathrm{ZnO}$ & & & & & & \\
\hline & INDUSTRIAL & - & -80.83 & -30.03 & -17.22 & -0.4 & -0.122 \\
\hline & SINTETIZADO & + & 80.83 & 30.03 & 17.22 & 0.4 & 0.122 \\
\hline \multirow[t]{3}{*}{2} & $\mathrm{SiO}_{2}$ & & & & & & \\
\hline & $15 \mathrm{phr}$ & - & -186.21 & -53.63 & -15.78 & -34.85 & 0.427 \\
\hline & $50 \mathrm{phr}$ & + & 186.21 & 53.64 & 15.78 & 34.84 & -0.427 \\
\hline \multirow[t]{3}{*}{3} & Interacción & & & & & & \\
\hline & $1 \& 2$ & - & -77.45 & -27.18 & -18.61 & -2.78 & 0.056 \\
\hline & & + & 77.45 & 27.19 & 18.61 & 2.78 & -0.056 \\
\hline
\end{tabular}


La resistencia a la abrasión es una propiedad importante en algunas aplicaciones del caucho, en cubiertas para autos por ejemplo, pero en muchas otras ella no es relevante y por lo tanto depende mucho de la naturaleza del servicio para la que es conformada la pieza. Realizado el análisis de los datos de este ensayo (10), se concluyó que el $\mathrm{SiO}_{2}$ contribuye apreciablemente a la abrasión no así el $\mathrm{ZnO}$, tal como se indica en la tabla 3.

\section{CONCLUSIONES}

Las principales conclusiones que se pueden extraer de este trabajo son las siguientes:

1.-) Las curvas de valoración potenciométrica y conductimétrica obtenidas, permiten determinar las etapas del proceso de síntesis que ocurren durante la adición del $\mathrm{NH}_{4} \mathrm{OH}$. La curva de valoración potenciométrica presenta tres (3) zonas bien definidas. A valores de $\mathrm{pH}$ bajos, zona I, la neutralización del ácido presente en el sistema es el principal fenómeno, acompañado de las reacciones de hidrólisis de las especies acuo presentes. En la zona II deben ocurrir, principalmente, reacciones de policondensación de las especies hidrolizadas y en la zona III formación de cincatos por la re-disolución del sólido precipitado y el alto consumo de base en el sistema.

2.-) Durante el proceso de síntesis, inicialmente en el sistema se deben formar complejos de cinc del tipo $\left[\mathrm{Zn}\left(\mathrm{NH}_{3}\right)_{\mathrm{n}}\right]\left(\mathrm{NO}_{3}\right)_{2^{\prime}},\left[\mathrm{ZnOH}\left(\mathrm{NH}_{3}\right)_{\mathrm{n}}\right]\left(\mathrm{CH}_{3} \mathrm{COO}\right),\left[\mathrm{Zn}(\mathrm{OH})_{2}\left(\mathrm{NH}_{3}\right)_{\mathrm{n}}\right]$ y $\left[\mathrm{ZnOH}\left(\mathrm{NH}_{3}\right)_{\mathrm{n}}\right]\left(\mathrm{NO}_{3}\right)$, entre otras. Estas especies mononucleares conformarían especies polinucleares que serían la base para que se constituyan especies sólidas cristalinas como $\mathrm{NH}_{4} \mathrm{Zn}_{5}(\mathrm{OH})_{9}\left(\mathrm{NO}_{3}\right)_{2} 3 \mathrm{H}_{2} \mathrm{O}, \mathrm{Zn}_{5}(\mathrm{OH})_{8}\left(\mathrm{CH}_{3} \mathrm{COO}\right)_{2}$. (2$\mathrm{x}) \mathrm{NH}_{3} \cdot \mathrm{xH}_{2} \mathrm{O}$ y $\mathrm{Zn}_{5}(\mathrm{OH})_{8}\left(\mathrm{NO}_{3}\right)_{2} \cdot(2-\mathrm{x}) \mathrm{NH}_{3} \cdot \mathrm{xH}_{2} \mathrm{O}$, tal como lo evidencian los resultados de DRX y FTIR.

3.-) Durante el proceso de lavado con agua, la fase sólida del sistema sufre transformaciones de fase conformándose principalmente $\mathrm{ZnO}$, cincita, siendo esta fase la más importante después de avanzado el proceso. El tamaño de las partículas obtenidas por MPC es nanométrico, $<200 \mathrm{~nm}$, y la microscopía electrónica de transmisión mostró que dichas partículas presentaban una morfología tipo aguja.

4-) El $\mathrm{ZnO}$ con morfología acicular obtenido del acetato de cinc, que se incorporó a la mezcla con la que se conforman los elastómeros, aporto propiedades reforzantes a la matriz, no tan importantes como el $\mathrm{SiO}_{2}$ pero sí lo suficientemente representativas como para seguir estudiándolo con otro tipo de elastómeros. Particularmente se puede destacar el efecto favorable del $\mathrm{ZnO}$ acicular sobre la resistencia a la tracción, elongación y módulo al $100 \%$.

\section{AGRADECIMIENTOS}

Este trabajo fue realizado en el marco del proyecto COLCIENCIAS 1103-08-12132. Un agradecimiento muy especial a la VRI de la universidad del Cauca por el apoyo brindado y a la Unidad de Microscopía Electrónica de la misma Universidad por la ayuda en los estudios con MET.

\section{REFERENCIAS}

1. A.S. Perl, Zinc oxide, Am, Ceram. Soc. Bull., 72, 6, 122-123 (1993)

2. M. Farnasworworth and C.H. Kline, Zinc Chemicals, International lead Zinc Research Org. Inc., New York., 243, (1973)

3. J. Livage, M. Henry, C. Sanchez. Sol-gel Chemistry of transition metal oxides, prog. Sold St. Chem., 18. 259-341 (1988).

4. J. A. Eduar, S. Sakk, C., Klein, L. Polymerized complex route to the synthesis of multicomponent oxides, sol-gel and technology. Ceramic Transactions (1995).

5. Li. Wen, ER Shi, Tian. Ming, The synthesis of $\mathrm{ZnO}$ acicular particles by the hydrotermal discharging-gas method, J. Mater. Res. 14, 4, 1532- 1537 (2003).

6. A. K. Vidal Pinilla, Determinación de compuestos intermediarios en el sistema $\mathrm{Zn}\left(\mathrm{CH}_{3} \mathrm{COO}\right)_{2}-\mathrm{NH}_{4} \mathrm{OH}-\mathrm{H}_{2} \mathrm{O}$ durante la síntesis de oxido de cinc por el método de precipitación controlada (MPC). Tesis de Grado Universidad del Cauca, (2003).

7. C. Ararat, J.A. Varela, J.E. Rodriguez Paez, Uso de métodos quimicos para obtener polvos cerámicos del sistema $(\mathrm{Sn}, \mathrm{Ti}) \mathrm{O}_{2}$. Bol. Soc. Esp. Ceram. V., 44, 4, 215-222 (2005)

8. J.E. Rodriguez - Paez, L.M. Ahumada, J.M. Bustamante, Sythesis of hydrated calcium silicate using the controlled precipitation method, Bol. Soc. Esp. Ceram. V., 44, 6, 421-426 (2005)

9. J.E. Rodriguez-Paez, A.C. Caballero, M. Villegas, controlled precipitation methods: formation mechanism of $\mathrm{ZnO}$ nanoparticles, J. Eur. Ceram. Soc., 21, 7, 925-930 (2001)

10. E.J. Andrade Campo, Síntesis de oxido de cinc $(\mathrm{ZnO})$ con morfología acicular para ser utilizado como refuerzo de elastómeros, Tesis de Grado Universidad del Cauca, (2005).

11. Robert o Babbit, The Vanderbilt handbook, Ed. R.T. Vanderbilt Company, (1978)

12. NORMAS ASTM (American society for testing and materials) 1990 annual book of ASTM standards session 9 rubber volumes 09.01 Rubber, Natural and synthetic- general test methods; carbon Black. (1990)

13. J.E. Rodríguez-Paez, Estudio de los mecanismos de formación de partículas de $\mathrm{ZnO}$ con diseño morfológico y dimensional obtenidas por el método de precipitación controlada, Tesis doctoral Universidad Autónoma de Madrid, (1999).

14. F.A. Cotton, y G. Wilkinson, Avanced inorganic chemistry. A comprehensive text, $5^{\circ}$. Ed., New York, Wiley-Interscience (1980).

15. A. Kawai, Y. Suga Hara, Preparation of zinc oxide Powders from 2dimensional hydroxide -zinc complexes of basic zinc acetate, chloride and nitrate, Ceramic powder science IV, Ceramic transactions Vol. 22, Ed. Hirano G. L. Messing, H. Hausner. The American Ceramic Society 75-80 (1991).

16. Nakamoto K., Infrared and Raman spectra of inorganic and coordination compounds, Part A, John Wiley \& sons, Inc, 5a edit. (1997).

17. S. Doeuff, M. Hehry, C. Sanchez Y J. Livage, Hydrolysis of Titanium alkoxides: modification of the molecular precursor by acetic acid. Journal of Non-crystalline solids, 5 206-216 (1987).

18. Ruiz Oscar A. Copolymer rubber \& Chemical. Design of experiments in the rubber industry. Rubber World. The technical service magazine for the rubber industry. 198 (1) 26-37 (1998).

Recibido: 23.11 .05

Aceptado: 27.04 .06 